\title{
Empleo público y Desgaste Profesional. Un Análisis desde las Características de la Tarea
}

\author{
Roxana Marsollier ${ }^{1}$
}

Recibido: 23/03/2017 Aceptado: 15/08/2017

DOI: 10.21772/ripo.v35n2a03

\begin{abstract}
Resumen
El presente artículo aborda la problemática del desgaste laboral en relación con las características de las tareas asociadas al empleo público. Se trabajó con una muestra intencional de 147 empleados que se desempeñan en una institución pública (Mendoza, Argentina). Se presentan los resultados del Maslach Burnout Inventory-General Survey (Schaufeli et al., 1996) y de una escala elaborada ad hoc sobre características del trabajo público. Los resultados alertan sobre los niveles de cansancio emocional y cinismo. En cuanto a las características de la tarea se analizaron indicadores tales como sobrecarga de trabajo, autonomía/motivación y aburrimiento/desventajas laborales. A nivel explicativo se comprobaron correlaciones y relaciones predictivas significativas entre las dimensiones del burnout y las características particulares de las tareas asignadas. Estos resultados constituyen un valioso aporte al estudio de la calidad de vida laboral y el perfil psicosocial del empleado estatal. A su vez, son una herramienta a considerar para la organización del trabajo, la prevención de riesgos psicosociales y la re-significación del empleo público.
\end{abstract}

Palabras clave: Desgaste laboral, Empleo Público, Características Laborales.

Public employment and Burnout. An analysis based on the characteristics of the job.

\begin{abstract}
This paper discusses burnout related to tasks associated with public employment. A deliberate sampling of 147 public employees from the government institution of Mendoza, Argentina, was used. The results shown arise from the Maslach Burnout InventoryGeneral Survey (Schaufeli et al., 1996) and from a chart developed ad hoc about the characteristics of public employment. The results are a warning concerning the levels of burnout and cynicism found among employees. Regarding the characteristics of the tasks, the factors analyzed were workload, autonomy / motivation and boredom / job disadvantages. At an explanatory level, there were significant, predictive co-relations and relations between the effects of burnout and the specific characteristics of the tasks assigned. These results are a valuable contribution to the study of the quality of working life and of the psychosocial profile of those working for the State. They are, in turn, a tool to be considered to organize tasks, prevent psychosocial risk and re-define public employment.
\end{abstract}

Key words: Burnout, Public Employment, Characteristics of the Job.

\footnotetext{
'Dra. en Educación. Consejo Nacional de Investigaciones Científicas y Técnicas (CONICET) y Universidad Nacional de Cuyo (UNCUYO), Mendoza, Argentina. e-mail: rgmarsollier@yahoo.com.ar

Nota: El presente artículo fue publicado en el 2017. Sin embargo, con el fin de mantener la periodicidad de la Revista, el número corresponde al segundo semestre del año 2016.

Cómo citar este artículo: Marsollier, R. (2016). Empleo Público y Desgaste Profesional. Un Análisis desde las Características de la Tarea. Revista Interamericana de Psicología Ocupacional, 35(2), 80-89. Doi: 10.21772/ripo.v35n2a03
} 


\section{Introducción}

En la actualidad, la investigación sobre los factores de riesgo psicosocial vinculados al trabajo cobra constantemente mayor relevancia en tanto, su prevención y tratamiento, tienen efectos importantes sobre la salud y bienestar de los trabajadores y, consecuentemente, en la salud y eficacia de las organizaciones (Peiró \& Rodríguez, 2008).

Cada contexto presenta características que le son propias y quienes se desempeñan en ellos, desarrollan una dinámica particular de trabajo. Así, cada ambiente laboral, se presenta como un escenario único y complejo, donde interactúan personas, grupos y la misma organización. Adentrarnos en la dimensión interior de cada organización para conocerla y poder identificar sus demandas y recursos laborales, requiere una mirada integral y comprehensiva de cada uno de sus elementos, procesos e interacciones.

En este estudio, buscamos conocer cómo son percibidas dichas demandas y recursos, así como el propio desempeño profesional en la dinámica peculiar de la organización, según la vivencia de cada trabajador estatal. Partimos del supuesto de que la autovaloración que tienen las personas sobre sí mismas, sus grupos de pertenencia y la estructura organizativa será condicionante de su bienestar o malestar en el trabajo. Desde esta óptica fijamos nuestro punto de abordaje.

Sin embargo, ¿por qué trabajar con el sector administrativo del empleo público? Pese a ser un grupo relegado en cuanto al estudio y tratamiento de riesgos psicosociales en el gobierno local, se trata de una población clave en el abordaje socio-político y laboral de la región. En nuestro contexto, los organismos estatales se hallan signados por representaciones sociales negativas del beneficio público y los procesos de modernización del Estado centran sus esfuerzos en otras áreas como las jurídicas y políticas, dejando en descuido a las personas que llevan adelante una empresa destinada al bien común. Por ello, en esta comunicación buscaremos aproximarnos a los condicionantes del desgaste profesional, especialmente, conocer cómo impacta la labor cotidiana en los trabajadores y si es posible que tal influencia pueda activar procesos psicosociales que lleven paulatinamente a cuadros de estrés o más aún, de burnout. En otros términos, nuestro objetivo es conocer si existe una vinculación significativa entre las características de las tareas puntuales que desempeña cada empleado y el desarrollo de los procesos de desgaste laboral en un contexto fuertemente burocratizado.

\section{Acerca del desgaste laboral}

Una de las consecuencias de la constante exposición a los riesgos psicosociales en el trabajo es el síndrome de burnout o desgaste profesional (Gil Monte, 2009). Se trata de una de las problemáticas más investigadas en los últimos 40 años a nivel mundial, por lo que es considerada un fenómeno transcultural (Gil Monte, 2007, Salanova \& Llorens, 2008).

El desgaste es concebido como un proceso que se desarrolla a partir del estrés crónico en el trabajo, fruto del agitado ritmo de vida actual (Salanova \& Llorens, 2011). Ya lo anticipaba Cherniss (1880) al considerar que el burnout es producto de una época signada por la sobrevaloración del éxito y la productividad, en desmedro de la persona humana. Tal es así, que este síndrome es considerado uno de los daños psicosociales más graves de nuestros tiempos (Salanova, 2006).

En el desarrollo del proceso conducente al desgaste profesional se van combinando variables personales, sociales y organizacionales que, a su vez, implican connotaciones negativas a nivel individual, grupal y laboral (Bresó, et al., 2007). Esta multiplicidad de factores intervinientes complejiza el abordaje y análisis del síndrome, y dan cuenta de la necesidad de recurrir a modelos integrales que permitan comprehender la dinámica que se da entre la persona y su entorno laboral.

En sus comienzos, el estudio del burnout se circunscribía a los servicios de ayuda, siendo abordado fundamentalmente en profesionales de la salud y la educación. Sin embargo, su investigación prontamente se extendió a otros perfiles laborales. Para poblaciones como la nuestra, denominadas de servicios generales, el desgaste laboral se manifiesta a través de elevados niveles de Cansancio emocional y de Cinismo, acompañados de una falta de Eficacia profesional (Maslach, Jackson \& Leiter, 1996). El Cansancio emocional alude a un agotamiento extremo, tanto a nivel emocional como físico que hace que la persona se sienta sobreexigida y desbordada por su situación. El cinismo, por su parte, se manifiesta como una respuesta insensible y negativa del sujeto hacia su tarea y contexto de trabajo (Maslach, 2009). El cinismo constituye una forma de despersonalización que pone en evidencia la desvalorización y el autosabotaje en relación con el valor del propio trabajo y también, de la organización (Moreno Jiménez, et al., 2001). Algunas investigaciones sostienen que el cinismo se encuentra asociado con estrategias de afrontamiento centradas en la emoción, que buscan el escape y la evitación de situaciones emocionalmente demandantes (Zamora et al., 2004). Estudios más recientes, identifican directamente este segundo constructo del burnout como distancia mental, incluyendo en esta noción tanto al cinismo -actitud distante hacia el propio trabajo- como a la despersonalización -actitud distante hacia las personas con las cuáles se comparte el trabajo cotidiano- (Salanova \& Llorens, 2011). Tanto el Cansancio emocional como el Cinismo -o despersonalización- constituyen las dimensiones más 
importantes del burnout, conocidas desde sus comienzos como el corazón del síndrome (Maslach \& Jackson, 1986).

Finalmente, la tercera dimensión alude a la Eficacia profesional. Es esperable que esta subescala puntúe en sentido inverso a las dos anteriores, por lo que podríamos decir que un cuadro de desgaste se caracterizaría por elevados niveles de Cansancio emocional y Cinismo y un sentimiento de baja Eficacia profesional. El sentimiento de ineficacia surge de la autoevaluación negativa del propio desempeño en el trabajo. En otros términos, quien no se siente eficiente, no confía en sus habilidades para realizar su trabajo de manera adecuada (Maslach, 2009, Salanova \& Llorens, 2011). Así, las consecuentes crisis de autoeficacia, causadas por reiteradas experiencias negativas de fracaso y falta de dominio, van fortaleciendo las creencias de ineficacia personal (Salanova, Lorente \& Vera, 2009).

\section{Características del empleo público y su relación con el burnout}

Hemos anticipado que cada ambiente de trabajo posee rasgos que le son propios y lo distinguen de cualquier otro ámbito laboral. Mediante el análisis de distintos estudios sobre el empleo público, procuramos identificar aquellas características de la tarea concreta que realizan los trabajadores en este ámbito, que lo distinguen de otro tipo de organizaciones y que potencialmente puedan considerarse como riesgos del trabajo estatal.

\section{Burocratización}

El primer aspecto a destacar, tiene que ver con la estructura de los organismos estatales, en particular el hecho de que se hallan fuertemente burocratizados. Nos referiremos entonces, a los procesos de burocratización, para indicar la disfuncionalidad del modelo burocrático weberiano que caracteriza, en general, a los organismos públicos. Las consecuencias de una estructura burocratizada afectan el comportamiento organizacional, en tanto los individuos y grupos se sienten limitados por un sistema autoritario y verticalista y no hallan un espacio propicio para su autonomía profesional. Así, la rigidez e impersonalización de las normas hace que los trabajadores perciban a la autoridad como arbitraria y sin contemplación de las diferencias individuales, imponiéndose como única meta el puro cumplimiento de las reglas (Pithod, 1993).

Las consecuencias negativas de esta estructura burocratizada impregnan y se manifiestan en el diseño de los puestos de trabajo. Así, aspectos tales como la rutina, la monotonía, la falta de autonomía en el trabajo se constituyen en verdaderos factores de riesgo psicosocial para estas poblaciones (Gil Monte \& Peiró, 1997, Marsollier, 2012) y podrían ser cruciales en los niveles de calidad organizacional. Estos riesgos, pueden convertirse en estresores que, de no ser afrontados apropiadamente, pueden desencadenar con el paso del tiempo, verdaderos procesos de desgaste. En este sentido, adquieren notabilidad el desarrollo y manejo de las estrategias de afrontamiento como un recurso personal de gran valía en el ámbito laboral (Martínez \& Salanova, 2009).

\section{Características de la tarea administrativa}

En este artículo nos centraremos en aquellos aspectos relacionados con la propia tarea, tal cual la percibe el empleado, dejando de lado otros aspectos como el clima laboral y las relaciones interpersonales. En otros términos, buscamos conocer si el trabajador advierte su tarea como agobiante o como desafiante, como aburrida o motivadora, es decir, qué significado adquiere para él.

Partimos del análisis efectuado por Gil Monte (2012), quien distingue diversos riesgos psicosociales que se hallan presentes en las características de la propia tarea, los aspectos organizacionales, las condiciones del empleo y la organización del tiempo de trabajo. Entre los riesgos vinculados a la propia tarea destaca la cantidad de trabajo, falta de autonomía, la monotonía, la ausencia de complejidad, entre otros aspectos. Sin embargo, no serán las características de la tarea, las que necesariamente impliquen un riesgo en sí mismas, sino que dependerá de cómo sean percibidas por los trabajadores (Schaufeli \& Salanova, 2002).

En relación con la carga de trabajo, Gil Monte, et al. (2008) comprobaron en sus estudios que una mayor carga de trabajo - ya sea cuantitativa o cualitativa- se asocia con el síndrome de burnout, en una muestra de profesiones de ayuda. Sin embargo, un trabajo efectuado por el Instituto Speyer de Alemania en 1999 sobre la productividad en la administración pública, concluyó que a la mayoría de los trabajadores les gustaría que se les aumentara la carga de trabajo, lo que estaría indicando que se sienten subvalorados en sus capacidades (Pérez Rubio \& Robina Ramírez, 2002).

Esto nos lleva al análisis de otra variable de interés: el tedio o aburrimiento en el trabajo. El tedio se despliega cuando la rutina y la monotonía predominan en el ambiente laboral e impactan negativamente en las personas (Pines \& Aronson, 1988). Al respecto, Moreno Jiménez, et al. (1997) consideran al aburrimiento como uno de los antecedentes del síndrome de burnout, conjuntamente con la realización de tareas que no son motivantes y otros aspectos disfuncionales de la organización. En un análisis en profundidad, Schaufeli y Salanova (2014) señalan que si bien se trata de problemáticas cuyas causas son diferentes, burnout (sobreestimulación) y aburrimiento o boredom (subestimulación), ambas comparten efectos similares tanto en las personas como en la organización, 
especialmente en cuanto a emociones y comportamientos negativos. Sin embargo, el aburrimiento en el trabajo es un factor poco abordado en los estudios de tipo psicosocial, siendo abundantes las aproximaciones efectuadas al modelo burnout-engagement.

Otro de los posibles factores en juego relacionados con la salud mental de los empleados es la ruptura del contrato psicológico. Dicho contrato, implica la percepción de ambas partes -individuo y organización- sobre el cumplimento del acuerdo, la confianza y la justicia (Gracia, et al., 2006).

Topa Cantisano y Morales (2005) señalan para una muestra de empleados del Estado español, que el burnout se pronostica a partir de determinadas condiciones del trabajo que terminan generando una ruptura del contrato psicológico, tales como la percepción de injusticia, el conflicto de rol y la falta de apoyo social. Aproximarnos a la noción de contrato psicológico nos lleva al análisis de su propio núcleo: las expectativas laborales, las que también cumplen un rol fundamental en el desarrollo del desgaste. Ya decía Pines (1993) que el burnout se desarrolla como un proceso de desilusión, cuando las expectativas no coinciden con lo que el medio laboral ofrece. Moreno Jiménez et al., (2009) analizan el rol de las expectativas en los procesos de desgaste encontrando asociaciones predictivas entre las expectativas de desarrollo profesional y los efectos negativos del burnout. Por su parte, Salanova, Lorente y Vera (2009) señalan que el modelo tradicional de contrato psicológico centrado en la estabilidad, ya no funciona en el mercado laboral actual, caracterizado por la flexibilidad y la globalización, lo que ocasionaría el quiebre del contrato psicológico con mayor facilidad.

\section{Metodología}

\section{Diseño y muestra}

Utilizamos un diseño metodológico mixto. De la instancia cuantitativa presentamos en esta comunicación los perfiles de desgaste laboral o burnout según las principales características autopercibidas de la tarea (administrativos del sector público).

Se trata de un estudio transversal, de muestreo intencional, conformado por personal administrativo que se desempeña en una dependencia gubernamental de la Provincia de Mendoza (Argentina). La muestra estuvo conformada por 147 empleados, el $48.3 \%$ de género femenino y el $51.7 \%$ masculino, de distintos niveles jerárquicos (jefes y no jefes) y condición laboral (planta permanente y contratados). La edad de los participantes oscila entre los 22 y 69 años, con un promedio de 43.88 años ( $\mathrm{DE}=11.95)$. La antigüedad es de 16.07 años promedio como empleado estatal $(\mathrm{DE}=10.95)$, con una media de 12.80 años ocupando el mismo puesto laboral (DE=10.90).

\section{Instrumentos}

Se aplicó un cuestionario semiestructurado destinado a recolectar variables de base (edad, sexo, antigüedad, nivel de instrucción, profesión, situación laboral, entre otras), a reconstruir la trayectoria laboral de los participantes y a abordar distintos factores mediante la aplicación de pruebas psicométricas. En este artículo, nos centramos en el resultado de dos de ellas:

a) El Maslach Burnout Inventory-General Survey (MBIGS) (Schaufeli, Leiter, Maslach y Jackson, 1996). Por tratarse de personal administrativo se utilizó esta versión del test que permite medir el desgaste laboral en personal de servicios generales. El MBI-GS contiene 16 ítems para evaluar sus tres dimensiones constitutivas: el Cansancio emocional (5 ítems, $\alpha=.826$, e. g., Trabajar todo el día realmente es estresante para mi); el Cinismo (5 ítems, $\alpha=.790$, e. g., Sólo quiero hacer mi trabajo y que no me molesten) y por último, la Eficacia profesional (6 ítems, $\alpha=.743$, e. g., $M e$ siento realizado cuando llevo a cabo algo en mi trabajo). Los participantes valoran cada ítem del cuestionario con una escala tipo Likert en la que indican la frecuencia con la que han experimentado la situación descrita en cada uno. Esta escala de frecuencia tiene siete grados que van de 0 ("Nunca") a 6 ("Todos los días"). Bajas puntuaciones en eficacia profesional y altos valores en cansancio emocional y cinismo suponen mayores niveles de desgaste. Cabe señalar, que el índice de confiabilidad calculado mediante Alpha de Cronbach corresponde a la muestra en estudio.

b) Características de la tarea: consiste en una escala de 16 ítems elaborada ad hoc centrada exclusivamente en los aspectos individuales de la propia tarea, tal como son autopercibidos por el participante, donde se valoran distintas apreciaciones del quehacer cotidiano en una escala Likert de 5 grados que van de 1 ("Totalmente en desacuerdo") a 5 ("Totalmente en acuerdo"). En cuanto a las cualidades psicométricas de la escala, se realizó un análisis factorial exploratorio con rotación Varimax. El índice de adecuación de la muestra $(\mathrm{KMO}=.708)$ y la prueba de esfericidad de Bartlett $\left(\chi^{2}\right.$ Bartlett $\left.=628,207, \mathrm{p}=.00\right)$ indican adecuados criterios empíricos para realizar el análisis. Así, se pueden identificar tres factores, que explican el $47,74 \%$ de la varianza total. El primer factor, alude a la Carga de trabajo (5 ítems, $\alpha=.746$, e. g., Realizo demasiadas tareas en función del horario laboral o Mi trabajo requiere mucha exigencia mental). El segundo factor se asocia a los niveles de Autonomía y motivación laboral (4 ítems, $\alpha=.634$, e. g., Puedo decidir cómo hacer mi trabajo o Mi trabajo es interesante y motivante). El tercer factor encontrado se refiere al Aburrimiento y Desventajas laborales (7 ítems, $\alpha=.606$, e. g., Mi trabajo me produce aburrimiento o El circuito burocrático entorpece el desarrollo de mi trabajo). 


\section{Procedimiento}

Los organismos públicos son instituciones de difícil acceso para la realización de proyectos de investigación, especialmente por la señalada burocratización del sistema y además, por tratarse de un estudio centrado en la calidad de vida laboral de los empleados. En este sentido, se observó cierta resistencia a participar del mismo.

Luego de conocer los fines de la investigación, quienes decidieron colaborar con el estudio, dejaron expresa constancia en un protocolo de consentimiento informado, alcanzando la muestra un $63 \%$ de la población que se desempeña como administrativo en la institución. La recolección de los datos se realizó en horario laboral, de manera personalizada, manteniendo el anonimato y bajo secreto estadístico. El procesamiento se efectuó mediante el software SPSS 19.0.0 en un tratamiento exhaustivo de los datos y en progresivos niveles de complejidad estadística.

\section{Resultados}

\section{Descriptivos según el MBI- GS}

Los resultados a nivel descriptivo señalan que la muestra, teniendo en cuenta sus puntuaciones promedio, no posee en su conjunto un perfil típico de burnout, ya que se mantienen elevados los niveles de eficacia profesional y los valores promedio para cansancio emocional y cinismo, son relativamente bajos, según los datos de la siguiente tabla (Ver tabla 1).

Tabla1 Valores descriptivos de las tres dimensiones de Burnout (escala 10)

\begin{tabular}{cccccc}
\hline Dimensión & Casos & Media & D.E.* & $\begin{array}{c}\text { Puntaje } \\
\text { mínimo }\end{array}$ & $\begin{array}{c}\text { Puntaje } \\
\text { máximo }\end{array}$ \\
\hline $\begin{array}{c}\text { Cansancio } \\
\text { emocional }\end{array}$ & 147 & 2.92 & 1.93 & 1 & 9 \\
\hline Cinismo & 147 & 2.22 & 1.83 & 0 & 9 \\
\hline $\begin{array}{c}\text { Eficacia } \\
\text { profesional }\end{array}$ & 147 & 7.52 & 1.31 & 2 & 9 \\
\hline * Desviación estándar & & & &
\end{tabular}

Fuente: Elaboración propia, a partir del análisis efectuado con el SPSS.

Sin embargo, al tratarse de una problemática tan delicada como es el desgaste, fue necesario profundizar en un análisis más detallado, así, según rangos del MBI, podemos vislumbrar tendencias más concretas (ver Tabla 2).
Tabla 2: Frecuencia absoluta y porcentaje por dimensión y rango (alto, medio, bajo) del MBI

\begin{tabular}{ccccc}
\hline Subescalas & Nivel & F & $\%$ & Acumulado \\
\hline \multirow{2}{*}{$\begin{array}{c}\text { Cansancio emocional } \\
\text { M=2.26 }\end{array}$} & Bajo & 45 & 30.6 & 30.60 \\
\cline { 2 - 5 } & Medio & 50 & 34.0 & 64.60 \\
\cline { 2 - 5 } & Alto & 52 & 35.4 & 100.00 \\
\hline \multirow{2}{*}{$\begin{array}{c}\text { Cinismo } \\
\text { M=2.92 }\end{array}$} & Bajo & 54 & 36.7 & 36.70 \\
\cline { 2 - 5 } & Alto & 47 & 32.0 & 100.00 \\
\hline \multirow{2}{*}{$\begin{array}{c}\text { Eficacia profesional } \\
\text { M=7.46 }\end{array}$} & Bajo & 49 & 33.3 & 33.30 \\
\cline { 2 - 5 } & Medio & 42 & 28.6 & 61.90 \\
\cline { 2 - 5 } & Alto & 56 & 38.0 & 100.00 \\
\hline
\end{tabular}

Fuente: Elaboración propia, a partir del análisis efectuado con el SPSS

Observamos que la distribución por porcentajes según los rangos bajo, medio y alto, es homogénea, ubicándose alrededor de un tercio de los participantes en niveles altos de cansancio emocional y cinismo y en similar proporción, de baja eficacia profesional, características típicas del síndrome. Estos datos son indicadores relevantes a tener en consideración, ya que estarían indicando la existencia de un sector de empleados que posee al menos alguna de las características propias del desgaste.

\section{Descriptivos de la escala sobre características de la tarea}

En cuanto a los resultados de nuestra prueba ad hoc sobre características de la tarea (especialmente, el trabajo administrativo que se desarrolla en los organismos públicos), se analizaron las tres subescalas identificadas en el análisis factorial: carga de trabajo, autonomía/motivación $\mathrm{y}$ aburrimiento/desventajas.

A fin de facilitar la interpretación de los resultados, dado el número disímil de indicadores de cada dimensión, para los análisis correspondientes se convirtieron todas las variables a escala 10, encontrando puntuaciones homogéneas.

La tabla 3 muestra los datos descriptivos de cada una de las dimensiones halladas. 
Tabla 3: Valores descriptivos de las tres dimensiones de Características de la tarea

\begin{tabular}{cccccc}
\hline Dimensión & Casos & Media & D.E.* & $\begin{array}{c}\text { Puntaje } \\
\text { mínimo }\end{array}$ & $\begin{array}{c}\text { Puntaje } \\
\text { máximo }\end{array}$ \\
\hline $\begin{array}{c}\text { Carga de } \\
\text { trabajo }\end{array}$ & 147 & 5.23 & 1.62 & 2 & 10 \\
\hline $\begin{array}{c}\text { Autonomía/ } \\
\text { motivación }\end{array}$ & 147 & 6.86 & 1.74 & 2 & 10 \\
\hline $\begin{array}{c}\text { Aburrimiento/ } \\
\text { desventajas }\end{array}$ & 147 & 4.49 & 1.27 & 2 & 8 \\
\hline \multicolumn{5}{c}{ * Desviación estándar }
\end{tabular}

Fuente: Elaboración propia, a partir del análisis efectuado con el SPSS.

Así, el factor que presenta mayor valor promedio es autonomía/motivación con una media de $6.86(\mathrm{DE}=1.74)$, sigue carga de trabajo con un promedio de $5.23(\mathrm{DE}=1.62)$ y finalmente, la dimensión vinculada al aburrimiento/ desventajas que presenta una media de $4.49(\mathrm{DE}=1.27)$.

La siguiente tabla muestra la distribución por rango de cada una de las dimensiones de la Escala sobre Características de la Tarea (Ver tabla 4).

Tabla 4: Frecuencia absoluta y porcentaje por dimensión y rango (bajo, medio, alto) de la Escala sobre Características de la tarea

\begin{tabular}{ccccc}
\hline Subescalas & Nivel & F & $\%$ & Acumulado \\
\hline \multirow{3}{*}{ Carga de trabajo } & Bajo & 54 & 36.7 & 36.7 \\
\cline { 2 - 5 } & Medio & 48 & 29.9 & 66.7 \\
\cline { 2 - 5 } & Alto & 49 & 33.3 & 100.0 \\
\hline \multirow{3}{*}{$\begin{array}{c}\text { Autonomía/ } \\
\text { Motivación }\end{array}$} & Bajo & 50 & 34.0 & 34.0 \\
\cline { 2 - 5 } & Medio & 42 & 28.6 & 62.6 \\
\cline { 2 - 5 } & Alto & 55 & 37.4 & 100.0 \\
\hline \multirow{3}{*}{$\begin{array}{c}\text { Aburrimiento/ } \\
\text { Desventajas }\end{array}$} & Bajo & 48 & 32.7 & 32.7 \\
\cline { 2 - 5 } & Medio & 55 & 37.4 & 70.1 \\
\hline
\end{tabular}

Fuente: Elaboración propia, a partir del análisis efectuado con el SPSS.

Como observamos, en nuestra muestra, los valores se distribuyen equitativamente entre los distintos rangos alto, medio y bajo, con porcentajes bastante similares. Se destacan algunos valores, por ejemplo, que el $36.7 \%$ de los encuestados consideran su carga de trabajo como baja, el $37.4 \%$ informan una alta autonomía/motivación en su tarea y en igual porcentaje, presentan un nivel medio de aburrimiento/desventajas laborales.

\section{Análisis bivariados}

En cuanto a los análisis bivariados, las dimensiones de desgaste profesional no presentaron asociaciones $\mathrm{o}$ correlaciones significativas con variables de base tales como género, estado civil, antigüedad o nivel de instrucción, etc.

En relación con las características individuales de la tarea, se observaron algunas correlaciones débiles con ciertas variables de base. Así, el factor carga laboral correlacionó con la obligación horaria $(\mathrm{r}=.162, \mathrm{p}<.005)$ y el Aburrimiento/Desventajas con la antigüedad como empleado estatal $(\mathrm{r}=.193, \mathrm{p}<.005)$.

En cuanto al análisis explicativo se observa que ambos instrumentos correlacionan entre sí, según se observa en la Tabla 5.

Tabla 5: Correlaciones entre el MBI-GS y la escala de Características de la tarea

\begin{tabular}{|c|c|c|c|c|c|c|c|}
\hline & & $\begin{array}{l}\text { Cansancio } \\
\text { Emocional }\end{array}$ & Cinismo & $\begin{array}{c}\text { Eficacia } \\
\text { Profesional }\end{array}$ & $\begin{array}{c}\text { Carga } \\
\text { Laboral }\end{array}$ & Autonomía & Aburrimiento \\
\hline \multirow[t]{3}{*}{$\begin{array}{l}\text { Cansancio } \\
\text { Emocional }\end{array}$} & $\begin{array}{l}\text { Correlación } \\
\text { de Pearson }\end{array}$ & 1 & $.508^{* *}$ & -.148 & $.209^{*}$ & $-.242^{* *}$ & $.250 * *$ \\
\hline & $\begin{array}{c}\text { Sig. } \\
\text { (bilateral) }\end{array}$ & & 0 & .073 & .011 & .003 & .002 \\
\hline & $\mathrm{N}$ & & 147 & 147 & 147 & 147 & 147 \\
\hline \multirow[t]{3}{*}{ Cinismo } & $\begin{array}{l}\text { Correlación } \\
\text { de Pearson }\end{array}$ & & 1 & $-.372^{* *}$ & -.032 & $-.369^{* *}$ & $.350 * *$ \\
\hline & $\begin{array}{c}\text { Sig. } \\
\text { (bilateral) }\end{array}$ & & & 0 & .699 & 0 & 0 \\
\hline & $\mathrm{N}$ & & & 147 & 147 & 147 & 147 \\
\hline \multirow[t]{3}{*}{$\begin{array}{c}\text { Eficacia } \\
\text { Profesional }\end{array}$} & $\begin{array}{l}\text { Correlación } \\
\text { de Pearson }\end{array}$ & & & 1 & $.180^{*}$ & $.442 * *$ & $-.204^{*}$ \\
\hline & $\begin{array}{l}\text { Sig. (bila- } \\
\text { teral) }\end{array}$ & & & & .029 & 0 & .013 \\
\hline & $\mathrm{N}$ & & & & 147 & 147 & 147 \\
\hline \multirow[t]{3}{*}{$\begin{array}{c}\text { Carga } \\
\text { Laboral }\end{array}$} & $\begin{array}{l}\text { Correlación } \\
\text { de Pearson }\end{array}$ & & & & 1 & .158 & .097 \\
\hline & $\begin{array}{c}\text { Sig. } \\
\text { (bilateral) }\end{array}$ & & & & & .056 & .241 \\
\hline & $\mathrm{N}$ & & & & & 147 & 147 \\
\hline \multirow[t]{3}{*}{ Autonomía } & $\begin{array}{l}\text { Correlación } \\
\text { de Pearson }\end{array}$ & & & & & 1 & $-.247^{* *}$ \\
\hline & $\begin{array}{c}\text { Sig. } \\
\text { (bilateral) }\end{array}$ & & & & & & .003 \\
\hline & $\mathrm{N}$ & & & & & & 147 \\
\hline $\begin{array}{l}\text { Aburri- } \\
\text { miento }\end{array}$ & $\begin{array}{l}\text { Correlación } \\
\text { de Pearson }\end{array}$ & & & & & & 1 \\
\hline
\end{tabular}

**. La correlación es significativa al nivel .01 (bilateral).

*. La correlación es significante al nivel .05 (bilateral)

Fuente: Elaboración propia, a partir del análisis efectuado con el SPSS..

Aunque las correlaciones que se identifican en la tabla anterior son significativas, sólo algunas superan el valor de .30 , correlación de magnitud pequeña $(\mathrm{r} \leq .30)$, según Aron y Aron (2002). Considerando este criterio, se destacan en relación con la dimensión Cinismo del MBI, una correlación positiva con el Aburrimiento/Desventajas $(\mathrm{r}=.350, \mathrm{p}<.001)$ y otra negativa con la Autonomía/Motivación $(r=-.369$, $\mathrm{p}<.001)$. 
Por otra parte, la Eficacia profesional, muestra particular correlación con el factor Autonomía/Motivación ( $\mathrm{r}=.442$, $\mathrm{p}<.001$ ), en todos los casos señalados, con un $99 \%$ de confianza.

\section{Análisis de regresión}

A nivel predictivo, los análisis de regresión lineal múltiple -según método de Pasos Sucesivos-, comprueban que si bien las asociaciones son significativas, el nivel predictivo es bajo, lo que estaría indicando que además de las características peculiares de la tarea realizada, existen otros factores de peso, que estarían mediando la relación con el desgaste profesional (Ver tabla 6).

Tabla 6: Modelo de Regresión lineal múltiple para Cansancio emocional, Cinismo y Eficacia profesional

\begin{tabular}{|c|c|c|c|c|c|}
\hline & \multicolumn{2}{|c|}{$\begin{array}{l}\text { Coeficientes no } \\
\text { estandarizados }\end{array}$} & \multicolumn{2}{|l|}{$\begin{array}{l}\text { Coeficientes } \\
\text { tipificados }\end{array}$} & \multirow[b]{2}{*}{ Sig. } \\
\hline & $\mathrm{B}$ & Error típ. & Beta & $\mathrm{t}$ & \\
\hline \multicolumn{6}{|c|}{ Modelo Cansancio Emocional $=\mathrm{R}^{2}=.147$} \\
\hline (Constante) & 2.120 & .974 & & 2.176 & .031 \\
\hline $\begin{array}{c}\text { Aburrimiento/ } \\
\text { Desventajas }\end{array}$ & .259 & .123 & .169 & 2.104 & .037 \\
\hline $\begin{array}{l}\text { Autonomía/ } \\
\text { Motivación }\end{array}$ & -.263 & .091 & -.236 & -2.906 & .004 \\
\hline Carga laboral & .275 & .095 & .229 & 2.902 & .004 \\
\hline \multicolumn{6}{|c|}{ Modelo Cinismo: $\mathrm{R}^{2}=.208$} \\
\hline (Constante) & 2.605 & .842 & & 3.093 & .002 \\
\hline $\begin{array}{l}\text { Autonomía/ } \\
\text { Motivación }\end{array}$ & -.317 & .081 & -.301 & -3.929 & .000 \\
\hline $\begin{array}{c}\text { Aburrimiento/ } \\
\text { Desventajas }\end{array}$ & .399 & .111 & .276 & 3.601 & .000 \\
\hline \multicolumn{6}{|c|}{ Modelo Eficacia profesional: $\mathrm{R}^{2}=.195$} \\
\hline (Constante) & 5.085 & .423 & & 12.027 & .000 \\
\hline $\begin{array}{l}\text { Autonomía/ } \\
\text { Motivación }\end{array}$ & .354 & .060 & .442 & 5.932 & .000 \\
\hline
\end{tabular}

Fuente: Elaboración propia, a partir del

análisis efectuado con el SPSS.

La tabla anterior muestra distintas relaciones predictivas entre las dimensiones de burnout y las características de la tarea. Así, tres variables explican el $14.7 \%$ de la variación del nivel de Cansancio emocional $\left(\mathrm{R}^{2}=.147\right)$ : a mayor Carga laboral $(\beta=.229, \mathrm{p}=.004)$ y Aburrimiento/Desventajas $(\beta=$ $.169, \mathrm{p}=.037)$ y menor nivel de Autonomía/Motivación $(\beta=$ $-.236, \mathrm{p}=.004)$, mayor Cansancio emocional.

En cuanto a la subescala de Cinismo, dos variables explican el $20,8 \%$ de la variación $\left(\mathrm{R}^{2}=.208\right)$ : un aumento en el nivel de Aburrimiento/Desventajas $(\beta=.276, \mathrm{p}=.000)$ y una menor Autonomía/Motivación $(\beta=-.301, \mathrm{p}=.000)$ predicen mayor nivel de Cinismo.

Finalmente, una variable, la Autonomía/Motivación ( $\beta=$ $.442, \mathrm{p}=.000)$ explica el $19.5 \%\left(\mathrm{R}^{2}=.195\right)$ de la variación en Eficacia profesional.

\section{Conclusión}

Los resultados obtenidos nos permiten una aproximación a cómo perciben los trabajadores estatales su propia tarea y cómo dicha autopercepción se relaciona con los procesos de desgaste laboral. Sin embargo, aunque no pueden ser generalizados, estos resultados son relevantes en el contexto de investigación, como aportes significativos para la toma de decisiones en los procesos de transformación del Estado a nivel local.

El recorrido efectuado permite observar que existe una asociación entre los factores abordados - burnout y características de la tarea-. Estadísticamente, los datos obtenidos son significativos y resultan incluso predictivos, aunque explican bajos porcentajes de la varianza. Ello indica que más allá de las características particulares de la tarea efectuada, existen otros factores de peso, que pueden representar verdaderas demandas para el sector.

La apreciación de una mayor carga laboral, los elevados niveles de aburrimiento y la percepción de una menor autonomía en el trabajo, han sido abordados en otras investigaciones sobre burnout, encontrándose coincidentemente vinculadas con el cansancio emocional. Estos factores son considerados en términos de demandas laborales que anteceden el desgaste (Cifre, Salanova \& Ventura, 2009).

Lo mismo ocurre con el cinismo, indicador común observado en poblaciones similares a la nuestra y factor clave en el desarrollo del desgaste laboral en profesiones de servicios generales (Moreno Jiménez, et al., 2001; Marsollier, 2009; Boechat \& Ferreira, 2014). Los resultados invitan a repensar el constructo cinismo, en tanto se encontraría asociado directamente con el aburrimiento y la falta de autonomía y, por ende, podría implicar una pérdida del significado de la propia tarea, aspectos que llevarían al trabajador a desarrollar esta reacción negativa frente a su trabajo.

Como era esperable, la percepción de autonomía/ motivación laboral se encontró asociada positivamente con los sentimientos de eficacia profesional, resultados todos en orden a la lógica y coherencia. Esta asociación positiva marca otro camino de abordaje psicosocial orientado a la salud ocupacional, destacando la importancia de fortalecer los recursos personales vinculados al trabajo y también a nivel organizacional (Salanova, Martínez, Cifre \& Llorens, 2009).

Las lecturas efectuadas nos permiten comprender mejor el comportamiento organizacional en el trabajo público, y la dinámica persona-contexto que subyace en organizaciones burocratizadas, en especial, al considerar que alrededor de un tercio de la muestra presenta claros indicadores de 
desgaste laboral. Ello, trae aparejadas consecuencias tanto personales como organizacionales.

Desde el punto de vista personal, es importante para estos empleados poder recuperar el sentido y significado del propio trabajo, entendiendo que si los mismos son construidos por los sujetos a partir de sus experiencias en la realidad (Da Rosa, et al., 2011) dichas experiencias deberían estar orientadas a un polo positivo, a optimizar el desarrollo tanto de las personas como de las instituciones.

Por su parte, desde la estructura organizativa, los resultados constituyen importantes insumos para la toma de decisiones vinculadas a los procesos de modernización y gestión estratégica de los recursos humanos, a fin de ser tenidos en cuenta en la reingeniería de los puestos de trabajo.

Los aspectos antes señalados, adquieren importancia desde el punto de vista formativo, ya que ponen de manifiesto la necesidad de desarrollar políticas de gestión que permitan trabajar desde la prevención, la intervención y la resignificación del trabajo público, tanto a nivel individual como organizacional y por qué no, a largo plazo, en la esfera comunitaria. Tal como afirma Pocoví (2009) el cambio cualitativo en la administración pública debe ser asumido por parte de todos los actores involucrados, ya sean funcionarios, empleados o ciudadanos. Por otra parte, señala esta autora, que los mismos empleados deben ser los protagonistas del cambio que se pretende, lo que puede lograrse mediante el desarrollo y gestión de los recursos humanos existentes y futuros. En otras palabras, el cambio será fruto de la misma transformación del Estado orientada al compromiso con la sociedad, en una gestión que priorice el bienestar de los ciudadanos, entendiendo que detrás de cada trámite o queja, hay una persona, una necesidad y un derecho (Blutman, 2009).

Finalmente, destacar que este estudio pretende ser para nuestro contexto, el puntapié inicial de un proceso de revisión de los riesgos psicosociales presentes en el sector público y de revalorización del trabajo estatal, cuya atención permita alcanzar mayores niveles de calidad de vida laboral para los empleados públicos y redunde en beneficio común.

\section{Referencias}

Aron, A., \& Aron, E. (2002). Estadística para Psicología. Buenos Aires: Pearson Education.

Blutman, G. (2009). La reforma y modernización del estado en Argentina: el papel de la cultura organizacional. Buenos Aires: Universidad de Buenos Aires, Facultad de Ciencias Económicas.

Boechat, M. \& Ferreira, M. (2014). Preditores individuais e organizacionais do burnout em servidores públicos federais. Psicologia, Saúde \& Doenças, 15(3), 738750 .

Bresó, E., Salanova, M., Schaufeli, W. \& Nogareda, C. (2007). Síndrome de estar quemado por el trabajo "Burnout" (III): Instrumento de medición. Nota Técnica de Prevención, 732, 21 a Serie. Instituto Nacional de Seguridad e Higiene en el trabajo.

Cherniss, C. (1980) Professional burnout in the human service organizations. New York: Praeger.

Cifre, E.; Salanova, M. \& Ventura, M. (2009). Demandas y recursos del ambiente de trabajo. En Salanova, M. Psicología de la Salud Ocupacional. Madrid: Síntesis.

Da Rosa Tolfo, S., Coutinho, M. C., Baasch, D., \& Cugnier, J. S. (2011). Sentidos y significados del trabajo: un análisis con base en diferentes perspectivas teóricas y epistemológicas en Psicología. Universitas Psychologica, 10(1), 175.

Gil Monte, P. \& Peiró, J. (1997). Desgaste psíquico en el trabajo. Madrid: Síntesis Psicología.

Gil Monte, P. (2007). El síndrome de quemarse por el trabajo (burnout) como fenómeno transcultural. Información Psicológica, (91-92), 4-11.

Gil Monte, P. (2012). Riesgos psicosociales en el trabajo y salud ocupacional. Revista Peruana de Medicina Experimental y Salud Pública, 29(2), 237-241.

Gil Monte, P. R., García-Juesas, J. A. \& Hernández, M. C. (2008). Influencia de la sobrecarga laboral y la autoeficacia sobre el síndrome de quemarse por el trabajo (burnout) en profesionales de enfermería. Journal of Psychology, 42(1), 113-118.

Gil-Monte, P. R. (2009). Algunas razones para considerar los riesgos psicosociales en el trabajo y sus consecuencias en la salud pública. Revista Española de salud pública, 83(2), 169-173.

Gracia, F. J., Silla, I., Peiró, J. M. \& Fortes-Ferreira, L. (2006). El estado del contrato psicológico y su relación con la salud psicológica de los empleados. Psicothema, 18(2), 256-262.

Marsollier, R. (2009) Educación y trabajo. Un estudio sobre factores educacionales y psicosociales que inciden en el desgaste laboral en la Administración Pública. Tesis para optar el título de Doctor en Educación, UNCUYO, Mendoza. Disponible en http://bdigital. uncu.edu.ar/5072.

Marsollier, R. (2012). El impacto de los factores organizacionales en el desgaste laboral. Un análisis 
en trabajadores estatales. Revista Interamericana de Psicología Ocupacional, 31 (1), 21-32.

Martínez, I. \& Salanova, M. (2009). Recursos personales: Inteligencia emocional y afrontamiento. En Salanova, M. Psicología de la Salud Ocupacional. Madrid: Síntesis.

Maslach, C. \& Jackson, S. (1986). The Burnout Research in the Social Services: a Critique. Special Issues: Burnout among Social Workers. Journal of Occupational Behaviour, 1, 95-105.

Maslach, C. (2009). Comprendiendo el burnout. Ciencia y Trabajo, 32: 37-43.

Maslach, C., Jackson, S. \& Leiter, M. (1996). Maslach Burnout Inventory Manual. Consulting Psychologists Press.

Moreno Jimenez, B., Rodríguez, R. \& Escobar, E. (2001). La evaluación del burnout profesional. Factorialización del MBI-GS. Un análisis preliminar. Ansiedad y Estrés, 7 (1), 69-78.

Moreno Jiménez, B., Rodríguez, R., Alvarez, A. \& Caballero, T. (1997). La evaluación del burnout: problemas y alternativas: el CBB como evaluación de los elementos del proceso. Revista de Psicología del Trabajo y las Organizaciones, 13 (2), 185-207.

Moreno Jiménez, B., Villa F, Rodríguez R. \& Villalpando J. (2009). Consecuencias Positivas y Negativas en el Trabajo: El Rol de las Expectativas Laborales En el Proceso de Desgaste Profesional. Ciencia y Trabajo. Abr-Jun, 11 (32): 80-84.

Peiró, J. M., \& Rodríguez, I. (2008). Estrés laboral, liderazgo y salud organizacional. Papeles del psicólogo, 29(1), 68-82.

Pérez Rubio, J. A. \& Robina Ramírez, R. (2002). Productividad y satisfacción laboral de los empleados públicos. El caso de Extremadura. Gestión y análisis de políticas públicas, (25), 101-108.

Pines, A. \& Aronson, E. (1988). Carreer burnout: causes and cures. Nueva York: Free Press.
Pines, A. (1993). Burnout: An existential perspective. En W.B. Schaufeli, C. Maslach \& T. Marek (Eds.), Profesional burnout: Recent developments in theory and research. Londres: Taylor y Francis, 35-51.

Pithod, A. (1993). Comportamiento organizacional. Psicología de las organizaciones. Buenos Aires: Editorial Docencia.

Pocoví, G. (2009). Gestión y desarrollo de recursos humanos: Clave para la transformación y la modernización de la administración pública. El caso de la Provincia de Santa Fe. Documentos y Aportes en Administración Pública y Gestión Estatal, (12), 101-104.

Salanova, M. \& Llorens, S. (2008). Estado actual y retos futuros en el estudio del burnout. Papeles del Psicólogo, 29 (1), 59-67.

Salanova, M. \& Llorens, S. (2011). Hacia una perspectiva psicosocial del "burnout": cuando el trabajo "nos" quema. En Agulló Tomás, E. Nuevas formas de organización del trabajo y la empleabilidad (pp. 271296). Universidad de Oviedo.

Salanova, M. (2006) Medida y evaluación del burnout, nuevas perspectivas. En Gil-Monte, P., Salanova, M., Aragón, J. \& Schaufeli, W. (eds.). El síndrome de quemarse por el trabajo en Servicios Sociales (pp. 27-43). Castelló de la Plana, Castellón, España: Universitat Jaume I

Salanova, M., Lorente, L. \& Vera, M. (2009). Demandas y recursos del ambiente de trabajo. En Salanova, M. Psicología de la Salud Ocupacional. Madrid: Síntesis.

Salanova, M., Martínez, I., Cifre, E. \& Llorens, S. (2009). Aspectos conceptuales de la Psicología de la Salud Ocupacional. En Salanova, M. Psicología de la Salud Ocupacional. Madrid: Síntesis.

Schaufeli, W. \& Salanova, M. (2002). ¿Cómo evaluar los riesgos psicosociales en el trabajo?. Prevención, Trabajo y Salud, 20, 4-9.

Schaufeli, W. \& Salanova, M. (2014). Burnout, boredom and engagement at the workplace. En M. Peeters, J. de Jonge \& T. Taris (Eds) People at work: An introduction to contemporary work psychology, 293320. 
Schaufeli, W., Leiter, M., Maslach, C., \& Jackson, S. (1996). The Maslach Burnout Inventory: General Survey (MBI-GS). En: Maslach, C., Jackson, C. \& Leiter, M. (Eds.). The Maslach Burnout Inventory Manual ( $3^{\text {a }}$ ed., pp. 19-26). Palo Alto, CA: Consulting Psychologists Press.

Topa Cantisano, G., Fernández Sedano, I. \& Lisbona Bañuelos, A. (2005). Ruptura de contrato psicológico y burnout en equipos de intervención en emergencias y catástrofes. Ansiedad y Estrés, 11(2-3), 265-279.

Zamora, G., Castejón, E. \& Fernández, I. (2004). Estar quemado (burnout) y su relación con el afrontamiento. International Journal of Clinical and Health Psychology, 4(2), 323-336. 